\title{
Developmental Changes in the Electrophysiologic Properties of Rabbit Papillary Muscles
}

\author{
TOSHIYUKI OSAKA, BRIAN M. RAMZA, ROSEMARIE C. TAN, AND RONALD W, JOYNER \\ Todd Franklin Cardiac Research Laboratory, Department of Pediatrics, Emory University School of Medicine, \\ Allanta, Georgia 30323
}

\begin{abstract}
We studied the electrophysiological properties of adult (AD) and newborn (NB) rabbit papillary muscles in vitro with superfusion of normal Tyrode's solution, solutions with elevated $\left[\mathrm{K}^{+}\right]_{0}$, and in solutions with various concentrations of tetrodotoxin. In control solutions, the NB papillary muscles had a more negative resting membrane potential $(-83.6 \pm 1.2$ versus $-80.0 \pm 1.5 \mathrm{mV})$, a higher rate of rise of phase $0(134 \pm 5$ versus $120 \pm 5$ $V / S$ ) and a higher, longer-lasting action potential plateau than the AD papillary muscles. Exposure to elevated $\left[\mathrm{K}^{+}\right]_{\mathrm{o}}$ led to a significant post-repolarization refractoriness in AD papillary muscles that was more than that for NB papillary muscles even when NB papillary muscles were depolarized to the same resting membrane potential as the AD papillary muscles. The NB papillary muscles were comparatively resistant to tetrodotoxin in terms of percent reduction of conduction velocity and percent rise in the current threshold for excitation. The conduction velocity for $A D$ papillary muscles in control solution $(66 \pm 6 \mathrm{~cm} / \mathrm{s})$ was more than for NB papillary muscles $(44 \pm 4 \mathrm{~cm} / \mathrm{s})$, which would not be expected from the data on the rate of rise of the action potential, suggesting that the cable properties of NB papillary muscles (specifically a greater surface to volume ratio of the ventricular cells) are also significantly different from the $\mathrm{AD}$ papillary muscles. ( $\mathrm{Pe}$ diatr Res 26: 000-000, 1989)
\end{abstract}

\section{Abbreviations}

RMP, resting membrane potential

$A P D$, action potential duration

APA, action potential amplitude

AD, adult

NB, newborn

TXX, tetrodotoxin

BE, bipolar electrode

The electrophysiologic properties of cardiac tissue have been shown to vary with the developmental age of the animal under normal conditions and with a variety of pharmacologic interventions. These age-related electrophysiologic differences have been shown to exist in the conduction system of the ventricle $(1,2)$ and in the atrioventricular node (3-7). With specific focus on the ventricular muscle, a developmental increase has been shown in the response to hypoxia in chick embryos $(8,9)$ and canine papillary muscles $(10)$. In response to hypoxia the functional

Received May 23, 1989; accepted July 28, 1989.

Correspondence Dr. R. W. Joyner. Todd Franklin Cardiac Research Laboratory, Department of Pediatrics, Emory University School of Medicine, Atlanta, GA 30323 .

Supported in part by NIH Grants HL22562 and HL41851 to R. W. J. and the Emory-Egleston Children`s Research Center. refractory period in AD papillary muscles became longer than the APD, accompanied by a significant RMP depolarization, whereas it required a much longer exposure to hypoxia in order to induce such a phenomenon in NB papillary muscles. We have observed a similar differential effect of hypoxia in rabbit papillary muscles.

Inasmuch as RMP depolarization decreases the $\mathrm{Na}^{+}$channel availability, one possible explanation for the age-related difference in ventricular refractoriness in response to hypoxia could be attributed to a difference in RMP between AD and NB resulting from the age-related changes in sensitivity to hypoxia of determinants maintaining RMP level, i.e., more $\mathrm{Na}^{+} / \mathrm{K}^{+}$ ATPase activity in newborn hearts $(1,11-13)$ or differential membrane permeability to potassium $(14,15)$. There have been some reports suggesting that $\mathrm{Na}^{+}$channel conductance in cardiac tissue also undergoes age-related changes, reflected by the agerelated difference in maximum rate of rise of action potential upstroke $\left(\dot{\mathrm{V}}_{\text {max }}\right)(2,16-19)$. Although there are discrepancies among species and tissues, the study by Cavoto et al. (16) showed that an age-related decrease in $\dot{V}_{\text {max }}$ in rat atrial muscles leads to another possibility that the difference in $\mathrm{Na}^{+}$channel availability in depolarized muscle with age may contribute to the age-related difference in refractoriness during hypoxia. Several studies have documented the effects of elevated $\left[\mathrm{K}^{+}\right]_{0}$ on adult ventricular tissue $(20-24)$. However, there have been no reports to examine the developmental changes in regard to the relationship between RMP level and the excitability of cardiac tissue.

The first purpose of the study was to compare the electrophysiologic properties of $A D$ and NB papillary muscles in normal $\left[\mathrm{K}^{+}\right]_{0}$ and to clarify the effect of potassium-induced RMP depolarization on the excitability and action potential properties of AD and NB papillary muscles through microelectrode recordings and programed stimulation.

It has been shown that the conduction velocity of the action potential depends on the cellular electrical properties and the properties of cellular interconnections and cellular geometry (25). The conduction velocity of the action potential varies directly as the square root of the maximum rate of rise of the action potential, but other factors are important in determining the conduction velocity. Conduction velocity has been shown to vary inversely with the square root of the surface to volume ratio $\left(S_{v}, \mathrm{~cm}^{-1}\right)$ and the square root of the longitudinal resistivity $\left(R_{a}\right.$, $\mathrm{ohm} \mathrm{cm}$ ). Thus, the second purpose of this study was to examine the developmental changes in the maximum rate of rise and the conduction velocity of the action potential in rabbit papillary muscles and to assess which of the above factors can account for the developmental differences.

\section{MATERIALS AND METHODS}

New Zealand White adult $(2-3 \mathrm{~kg})$ and neonatal (1-3 d) rabbits of either sex were used in the experiments. The rabbits were anesthetized with sodium pentobarbital $(50 \mathrm{mg} / \mathrm{kg}$ intravenous for adults and intraperitoneal for newborns). The hearts were 
quickly excised via a thoracotomy and placed in a beaker of oxygenated Tyrode's solution with $\mathrm{mM}$ composition $\mathrm{NaCl} 120.3$, $\mathrm{NaH}_{2} \mathrm{PO}_{4}$ 1.2, $\mathrm{NaHCO}_{3} 24.2, \mathrm{KCl} 4, \mathrm{MgCl}_{2} 1.3, \mathrm{CaCl}_{2} 1.2$, and dextrose 5.5. The left ventricle was opened and left papillary muscles removed with some attached ventricular wall and placed in a lucite chamber with the above Tyrode's solution at $36^{\circ} \mathrm{C}$, continuously aerated with $95 \% \mathrm{O}_{2}-5 \% \mathrm{CO}_{2}, \mathrm{pH} 7.35-7.4$. Data from a total of $13 \mathrm{AD}$ papillary muscles and $16 \mathrm{NB}$ papillary muscles in which the interventions of elevated potassium or application of TTX were reversible are included in our results.

Extracellular recording and stimulating electrodes were made from a twisted pair of Teflon-coated silver wires with a wire diameter of $75 \mu$. Microelectrodes were filled with $2.5 \mathrm{M} \mathrm{KCl}$ and had resistances of 20-25 Mohms. To test the electrophysiologic properties of $\mathrm{AD}$ and NB papillary muscles, we used an electrode arrangement as in Figure 1. Two bipolar surface electrodes (S1, S2) were used as stimulators. The S1 stimulator was used to deliver a train of 10 stimuli at a basic cycle length of 500 $\mathrm{ms}$, duration $2 \mathrm{~ms}$, with a stimulus intensity of 1.5 times the excitation threshold. After each train of $10 \mathrm{SI}$ stimuli, a stimulus was applied through the $\$ 2$ bipolar electrode with a variable delay after the 10 th $S 1$ stimulus. A microelectrode was placed as near as possible (within $0.25 \mathrm{~mm}$ ) of the $S 2$ electrode to continuously record the intracellular potential. Thus, for each $S 1$ stimulus the microelectrode records a propagating action potential and for each $\$ 2$ stimulus the microelectrode records the local response. We placed a third bipolar electrode several millimeters away from the $\mathrm{S} 2$ location. This BE measured the surface potential waveform. A successful S1 or S2 stimulus caused a biphasic surface electrogram at the BE recording electrode. For experiments in which the conduction velocity was measured, we used two $\mathrm{BE}$ electrodes separated by $1 \mathrm{~mm}$ along the long axis of the papillary muscle to measure conduction time per mm. Tetrodotoxin (Sigma Chemical Co., St. Louis, MO) was used at concentrations from $1-5 \mu \mathrm{M}$.

For each papillary muscle, we used the following protocol in control conditions $\left(\left[\mathrm{K}^{+}\right]_{0}=4 \mathrm{mM}\right)$, a test solution $\left(\left[\mathrm{K}^{+}\right]_{\mathrm{o}}=8\right.$ or $9 \mathrm{mM})$, and in recovery $\left(\left[\mathrm{K}^{+}\right]_{0}=4 \mathrm{mM}\right)$ with a single sustained microelectrode penetration. We first determined the $\mathrm{S} 2$ current threshold (I2) for successful S2 stimulation with a long delay ( $\mathrm{S} 1-\mathrm{S} 2$ interval equal to $500 \mathrm{~ms}$ ) between the 10th $\mathrm{S} 1$ and the S2 stimulus. We then raised $I 2$ by $25 \%$ and gradually reduced the $\$ 1-\$ 2$ interval until the $\$ 2$ stimulus was unsuccessful at generating a propagating action potential. We repeated this process with increases of $50,100,150$, and $200 \%$ in 12 , finding, for each value of 12 , the shortest $\$ 1-\$ 2$ interval for successful stimulation of a propagated $\$ 2$ response.

Statistical analysis was done by multiple $t$-tests, with the protocols arranged such that each potassium concentration or tetrodotoxin concentration tested had a separate control period in each preparation.

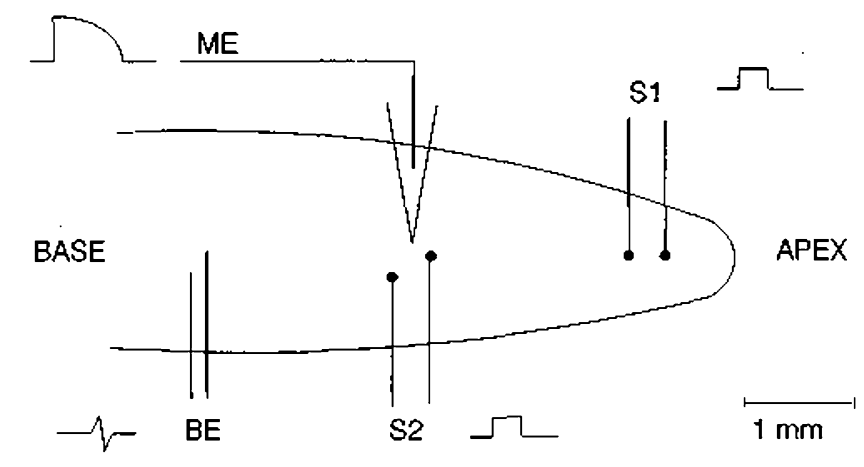

Fig. 1. A diagram of the papillary muscle preparation, showing the locations of the $S 1$ and $S 2$ stimulating electrodes and the microelectrode $(M E)$ and bipolar $(B E)$ recording electrodes.

\section{RESULTS}

Figure 2 shows data from an AD papillary muscle in 4 and 8 $\mathrm{mM}\left[\mathrm{K}^{+}\right]_{\text {. }}$. Each trace is the microelectrode recording starting just before the 10th $S 1$ response and continuing through the $\mathrm{S} 2$ response. For both $4 \mathrm{mM}\left[\mathrm{K}^{+}\right]_{0}$ (upper trace) and $8 \mathrm{mM}\left[\mathrm{K}^{+}\right]_{0}$ (lower trace) we show the recordings made for the shortest $\mathrm{S} 1$ S2 interval at which an I2 of two times the diastolic threshold was successful in generating a propagating action potential.

Several features of the $S 1$ action potential from eight $A D$ papillary muscles are summarized in Table 1 for $\left[\mathrm{K}^{+}\right]_{0}=4$ or 8 $\mathrm{mM}$. The RMP was $-80.0 \pm 1.5 \mathrm{mV}$ (mean $\pm \mathrm{SD})$. We measured the APD at two different voltage levels. We defined APD $(0 \mathrm{mV})$ as the time from the maximum upstroke velocity to the time of repolarization to just below $0 \mathrm{mV}$. We also define APD (RMP $+5 \mathrm{mV}$ ) as the time from the maximum upstroke velocity to the time of repolarization to within $5 \mathrm{mV}$ of the RMP. We have used these measurements instead of the more conventional measurements of time to 50 or $95 \%$ repolarization for two reasons. First, the conventional measurement parameters define a duration that depends on both the resting potential and on the spike amplitude, and both of these parameters are changed by our interventions. Second, we wanted to emphasize the effects of membrane potential on the recovery of excitability, and thus wanted to define a repolarization time to within a constant voltage level $(5 \mathrm{mV})$ of the resting potential for the control and the intervention solutions. On the upper and lower traces of Figure 2 we have drawn horizontal dashed lines for the voltage levels RMP $+5 \mathrm{mV}$. for each trace. Note that this level changes with the RMP as the papillary muscle is depolarized with $8 \mathrm{mM}$ $\left[\mathrm{K}^{+}\right]_{\mathrm{o}}$. The time at which repolarization has occurred to within $5 \mathrm{mV}$ of the RMP (indicated by the arrows in Figure 2) is also used as a zero time reference to express the degree of prematurity of the $\$ 2$ stimulus. We define Stimulus Delay (SD) as the time of the $S 2$ stimulus with respect to the time at which repolarization to within $5 \mathrm{mV}$ of the RMP has occurred. One feature that becomes very apparent with this definition of SD time is that one effect of the $8 \mathrm{mM}\left[\mathrm{K}^{+}\right]_{0}$ is to produce postrepolarization refractoriness. Table 1 shows that the SD times for successful stimulation with $\mathrm{I} 2$ at two times the diastolic threshold was -2.0 $\pm 1.3 \mathrm{~ms}$ for $\left[\mathrm{K}^{+}\right]_{0}=4 \mathrm{mM}$ and was $25.5 \pm 9 \mathrm{~ms}$ for $\left[\mathrm{K}^{+}\right]_{0}=8$ $\mathrm{mM}$ for AD papillary muscles. Another effect of elevating $\left[\mathrm{K}^{+}\right]_{0}$

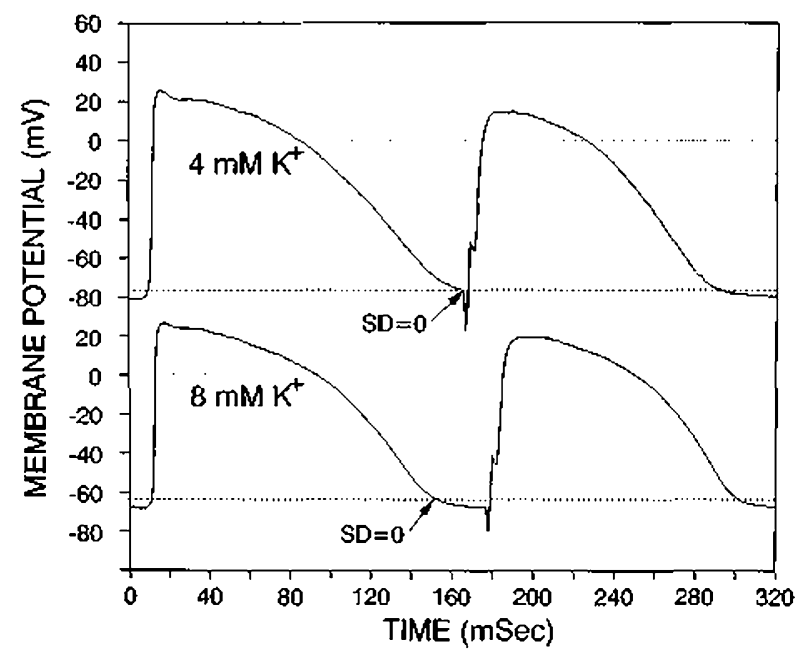

Fig. 2. A comparison of the adult action potentials in 4 and $8 \mathrm{mM}$ $\left[\mathrm{K}^{+}\right]_{0}$. Each trace begins with the $10^{\text {th }}$ action potential stimulated from the $\mathrm{SI}$ electrode. For each value of $\left[\mathrm{K}^{+}\right]_{0}$ we first determined the diastolic threshold for the $\$ 2$ stimulus, then doubled the $\$ 2$ stimulus and recorded the traces shown using the earliest SI-S2 interval for successful S2 action potential propagation. The time marked " $\mathrm{SD}=0^{\text {n }}$ on each trace corresponds to the time at which repolarization had progressed to within 5 $\mathrm{mV}$ of the RMP. 